\title{
Isolation and Characterization of 10 Polymorphic Microsatellite Loci in Paphiopedilum concolor (Batem.) Pfitzer (Orchidaceae) and Cross-species Amplification
}

\author{
Long-na Li and Songjun Zeng ${ }^{1}$
}

Key Laboratory of Plant Resources Conservation and Sustainable Utilization, South China Botanical Garden, Chinese Academy of Sciences, Xingkelu 723, Tianhe, Guangzhou, Guangdong 510650, China; and the Graduate University of Chinese Academy of Sciences, Beijing 100039, China

\section{Feng Zheng}

Key Laboratory of Plant Resources Conservation and Sustainable Utilization, South China Botanical Garden, Chinese Academy of Sciences, Xingkelu 723, Tianhe, Guangzhou, Guangdong 510650, China

\section{Zhi-lin Chen}

Key Laboratory of Plant Resources Conservation and Sustainable Utilization, South China Botanical Garden, Chinese Academy of Sciences, Guangzhou 510650, China; and Horticultural Research Institute of Guizhou Province, Guiyang 550006, China

\section{Kun-lin Wu, Jian-xia Zhang, and June Duan}

Key Laboratory of Plant Resources Conservation and Sustainable Utilization, South China Botanical Garden, Chinese Academy of Sciences, Guangzhou 510650, China

Additional index words. Paphiopedilum concolor, microsatellite, cross-species amplification, Orchidaceae

\begin{abstract}
Ten polymorphic microsatellite loci were isolated and characterized from an enriched genomic library of Paphiopedilum concolor (Batem.) Pfitzer. The number of alleles per microsatellite locus ranged from three to 11 with an average of 6.4 in a sample of 30 individuals from three populations. The observed and expected heterozygosity ranged from 0.200 to 0.800 and from 0.544 to 0.827 , respectively. These microsatellites can be used as tools to investigate the genetic structure of $P$. concolor populations and relationship patterns with closely related taxa.
\end{abstract}

The genus Paphiopedilum Pfitzer, popularly known as slipper orchids because of the resemblance of the pouch-shaped lip to a lady's slipper (Cribb, 1998; Sheehan and

\footnotetext{
Received for publication 13 Apr. 2010. Accepted for publication 12 May 2010.

The study was supported by National Key Project of Scientific and Technical Supporting Programs Funded by the Ministry of Science and Technology of China (No. 2008BAC39B05) and Guangdong Key Technology Research and Development Program (No. 2009B020201009).

We are grateful to Kuai-fei Xia and Zheng-feng Wang of South China Botany Garden for their assistance during experimentation and manuscript preparation.

The first two authors contributed equally to this work. ${ }^{1}$ To whom reprint requests should be addressed; e-mailduanj@scib.ac.cn.
}

Sheehan, 1994), have captured the interest of many orchid growers and hobbyists, and Paphiopedilum are one of the most popular and rare orchid genera being sold and exhibited today. Their unique shape, variety of colors, and extended flowering period make them distinctive to the orchid family. The genus is comprised of $\approx 77$ species found in southern India, Nepal, Bhutan, northeast India, and Burma across to southern China, Southeast Asia, the Malay Archipelago, the Philippines, New Guinea, and the Solomon Islands. A total of 27 species of Paphiopedilum have been reported to be present in China (Cribb, 1998; Liu et al., 2009a, 2009 b). Wild populations are under increasing pressure from overcollection and habitat destruction, and all species are listed in the Convention on International Trade in Endangered Species of Wild Fauna and Flora
(CITES), Appendix I, thus restricting trade of these plants.

Like many other slipper orchids, $P$. concolor (Batem.) Pfitzer, a native of southeast Burma, southwest China (Yunan, Guizhou, and Guangxi provinces), Thailand, Laos, Cambodia, and Vietnam, has become a popular ornamental plant since its discovery in 1865 (Cribb, 1998; Liu et al., 2009a). This popularity as well as general habitat destruction and overcollection, has contributed to a drastic decline in the number and range of the wild populations. Determining the genetic diversity of the remaining wild plants is a critical step in developing effective conservation strategies for $P$. concolor as well as many other species. Microsatellites are ideal markers for characterizing of population structure (Schlotterer, 1998) and have previously been used for the study of endangered orchids (Rodrigues and Kumar, 2009). In the current investigation, we focused on microsatellite isolation in $P$. concolor to obtain polymorphic markers for investigation of population genetics.

A microsatellite-enhanced genomic library was constructed using an optimized affinity capture technique described by Fleischer and Loew (1995) and Xia et al. (2006). A P. concolor plant was sampled from Yunnan province, China. Genomic DNA was extracted from fresh leaf tissue by using the cetyltrimethyl ammonium bromide method (Doyle, 1991). We constructed an enriched partial genomic library following a modified protocol of fast isolation by amplified fragment length polymorphism of sequences containing repeats (FIASCO) (Zane et al., 2002). Approximately $250 \mathrm{ng}$ of genomic DNA was digested into 200 to $1000 \mathrm{bp}$ by a restriction enzyme $\mathrm{MboI}$ (TaKaRa, Japan) and the resulting fragments were ligated to $\mathrm{MboI}$ linkers (SAULA: 5'G CGGTACCCGGGAAGCTTGG3'; SAULB: 5' GATCCCAAGCTTCCCGGGTACCGC3') with T4 DNA ligase (TaKaRa) and incubated overnight at $16{ }^{\circ} \mathrm{C}$. The digestion-ligation mixture was subsequently diluted 10 times, and $2 \mu \mathrm{L}$ was used for polymerase chain reaction (PCR) amplification with SAULA oligonucleotide as the primer. PCR products were hybridized to two single-strand biotinylated microsatellite oligonucleotides $\left[(\mathrm{CA})_{12^{-}}\right.$ biotion and $(\mathrm{GA})_{12}$-biotin]. These hybrids were captured by streptavidin-coated magnetic beads (BioMag Nuclease-Free Streptavidin; Qiagen). The magnetic beads were eluted and the resulting DNA solution served as a template for PCR using SAULA olignucleotide as the primer. The amplification product was directly ligated into the pGEM-T plasmid vector (Promega, Madison, WI) and transformed into Escherichia coli. DH5 $\alpha$ competent cells $(\mathrm{TaKaRa})$ were plated onto LB agar medium (Bacto-Tryptone $10 \mathrm{~g} \cdot \mathrm{L}^{-1}$, Bactoyeast extract $5 \mathrm{~g} \cdot \mathrm{L}^{-1}, \mathrm{NaCl} 5 \mathrm{~g} \cdot \mathrm{L}^{-1}$, agar $\left.6 \mathrm{~g} \cdot \mathrm{L}^{-1}\right)$. Recombinant plasmids were identified through blue-white screening. For further identification of the clones containing microsatellites, colony PCR was performed with a primer combination of $(\mathrm{CA})_{12}$ or $(\mathrm{GA})_{12}$ and M13 universal primers. The insert fragments 
Table 1. Characteristics of 10 microsatellite loci for P. concolor

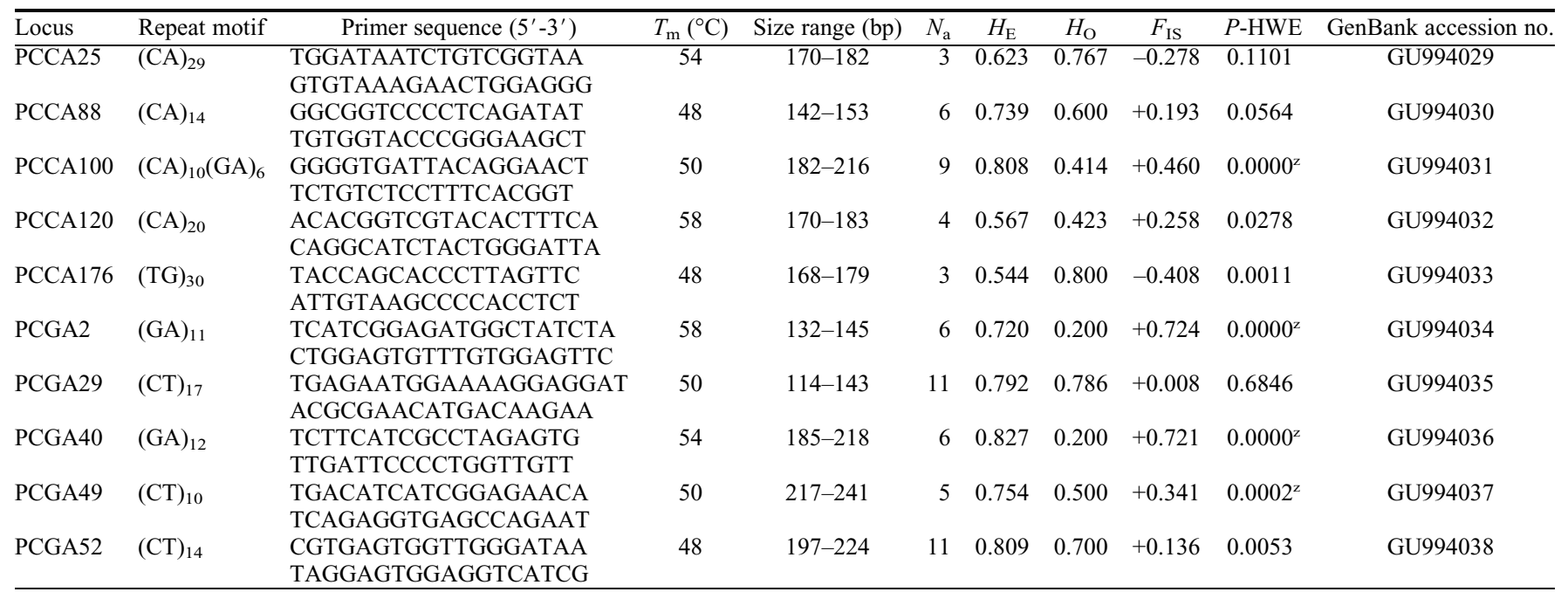

${ }^{\mathrm{z}}$ Significant deviation from Hardy-Weinberg equilibrium corrected for multiple comparisons using the Bonferroni correction $(P<0.001)$.

$T_{\mathrm{m}}=$ annealing temperature; $N_{\mathrm{a}}=$ number of alleles; $H_{\mathrm{E}}=$ expected heterozygosity; $H_{\mathrm{o}}=$ observed heterozygosity; $F_{\mathrm{IS}}=$ inbreeding coefficient; $P$-HWE $=$ probability of Hardy-Weinberg equilibrium.

of 124 positive clones were sequenced. Among the $97(78.23 \%)$ positive clones containing microsatellites with five or more repeat units, 63 were selected for primer development. Amplification primers were designed using PRIMER 5.0. To control for polymorphism of the identified microsatellite loci, 30 individual $P$. concolor were used from three natural populations in Baoshan and Wenshan in Yunnan province and Pengxiang in Guangxi province and planted in a greenhouse in South China Botanical Garden, Chinese Academy of Sciences. PCR amplification was performed in $10-\mu \mathrm{L}$ reaction mixtures consisting of $\approx 50 \mathrm{ng}$ of template DNA, $50 \mathrm{~mm}$ of $\mathrm{KCl}, 20 \mathrm{~mm}$ of Tris- $\mathrm{HCl}(8.0), 1.5 \mathrm{~mm}$ of $\mathrm{MgCl}_{2}, 0.2 \mathrm{~mm}$ of each dNTP, $0.2 \mu \mathrm{M}$ of each primer, and $1 \mathrm{U}$ of Taq DNA polymerase (TaKaRa). The reaction mixture was subjected to PCR amplification in a PTC-100 (MJ) with the following cycling profile: $5 \mathrm{~min}$ at $95^{\circ} \mathrm{C}$ followed by 35 cycles of $40 \mathrm{~s}$ at $94{ }^{\circ} \mathrm{C}, 50 \mathrm{~s}$ at the primer specific annealing temperature (Table 1 ), 1 min at $72{ }^{\circ} \mathrm{C}$, and a final prolonged extension of 10 min at $72{ }^{\circ} \mathrm{C}$. PCR products were separated on $5 \%$ denaturing polyacrylamide gels using a 100-bp DNA ladder standard (Promega) as the reference and visualized by silver staining. The microsatellite alleles were sized by using LABIMAGE Version 2.7.1.

Approximately 47 of the 63 primer pairs were successfully amplified containing 10 polymorphic loci and 37 monomorphic loci. Variability of the microsatellite loci was evaluated using POPGENE32 (Yeh et al., 1997). Deviation from Hardy-Weinberg equilibrium (HWE) and linkage disequilibrium were tested with GENEPOP Version 3.4 (Raymond and Rousset, 1995) and Rice's method of sequential Bonferroni correction (Rice, 1989).

Genotyping of all individuals revealed moderately high polymorphism with three to 12 alleles per locus and an across-loci average of 6.4 alleles per locus. The expected heterozygosity ranged from 0.200 to 0.800 , and the observed heterozygosity varied from 0.544 to 0.827 (Table 1), respectively. No evidence of linkage disequilibrium was found between any loci pairs, indicating physical independence of the 10 loci. Four loci, PCCA100, PCGA2, PCGA40, and PCGA49, exhibited significant deviation from HWE after Bonferroni correction (adjusted $P$ value $=$ 0.001 ; Table 1) as a result of heterozygote deficiency (positive $F_{\text {IS }}$ values), which could be caused by inbreeding in this species.

We also tested for cross-species amplification of the $10 P$. concolor microsatellite primers in two closely related species, $P$. bellatulum (Rchb.f.) Pfitzer and $P$. wenshanense Z.J.Liu \& J.Yong Zhang (Liu et al., 2009a, 2009b) using the same PCR conditions as previously described. Seven positive amplifications occurred across all tested species; the remaining three (PCCA88, PCCA100, and PCCA176) resulted in weak amplifications.

Data generated in this study strongly indicate that these newly developed microsatellite loci are potentially useful markers for investigating both population genetics and conservation biology of the congenetic Paphiopedilum species. The results have also great potential value for identification of hybrids of this slipper orchid, for analyzing genetic structure of native populations, and for learning the genetic background of the artificial growing individuals for restoring wild populations of this slipper orchid.

\section{Literature Cited}

Cribb, P. 1998. The Genus Paphiopedilum. 2nd Ed. National History Publications, Borneo, Malaysia

Doyle, J.J. 1991. DNA protocols for plants-CTAB total DNA isolation, p. 283-293. In: Hewitt,
G.M. and A. Johnston (eds.). Molecular techniques in taxonomy. Springer-Verlag, Berlin, Germany.

Fleischer, R.C. and S. Loew. 1995. Conservation and screening of microsatellite-enriched genomic libraries, p. 459-468. In: Ferraris, J. and S. Palumbi (eds.). Molecular zoology: Advances, strategies and protocols. Wiley-Liss, New York, NY.

Liu, Z.J., S.C. Chen, L.J. Chen, and S.P. Lei. 2009a. The genus Paphiopedilum in China. Science Press, Beijing, China.

Liu, Z.J., X.Q. Chen, and P.J. Cribb. 2009b. Paphiopedilum, p. 33-34. In: Wu, Z.Y., P.H. Raven, and D.Y. Hong (eds.). Flora of China. Vol. 25 (Orchidaceae). Science Press, Beijing, China, and Missouri Botanical Garden Press, St. Louis, MO.

Raymond, M. and F. Rousset. 1995. GENEPOP (Version 1.2): Population genetics software for exact tests and ecumenicism. J. Hered. 86:248249.

Rice, W.R. 1989. Analyzing tables of statistical tests. Evolution 43:223-225.

Rodrigues, K.F. and S.V. Kumar. 2009. Isolation and characterization of microsatellite loci in Phalaenopsis gigantean. Conserv. Genet 10: 559-562.

Schlotterer, C. 1998. Microsatellites, p. 237-261. In: Hoelzel, A. (ed.). Molecular genetic analysis of populations: A practical approach. Oxford University Press, Oxford, UK.

Sheehan, T.J. and M. Sheehan. 1994. An illustrated survey of orchid genera. Timber Press Inc., Portland, OR

Xia, J.H., K.F. Xia, and S.G. Jiang. 2006. Characterization of 11 polymorphic microsatellite loci in the yellowfin seabream Acanthopagrus latus. Mol. Ecol. Notes 6:484-486.

Yeh, F.C., R.C. Yang, T.B.J. Boyle, Z.H. Ye, and J.X. Mao. 1997. POPGENE, the user-friendly shareware for population genetic analysis. Molecular Biology and Biotechnology Centre, University of Alberta, Alberta, Canada.

Zane, L., L. Bargelloni, and T. Patarnello. 2002. Strategies for microsatellite isolation: A review. Mol. Ecol 11:1-16. 